\title{
BreastCare
}

\section{If Chemotherapy Is Indicated, Give the Optimal Regimen!}

\author{
Volker Möbus \\ Klinik für Gynäkologie und Geburtshilfe, Klinikum Frankfurt Höchst, Frankfurt/M., Germany
}

The 5-year survival data of breast cancer patients have reached outstanding results. Nitz et al. [1] reported a 5-year overall survival rate for the formerly called 'intermediate risk group' (patients with 1-3 positive lymph nodes) of $95 \%$, achieved by a modern anthracycline/taxane containing regimen of 4 cycles epirubicin + cyclophosphamide followed by 4 cycles docetaxel. In a combined analysis of 2 intense dose-dense trials Thomssen et al. [2] have previously reported a 5 -year overall survival rate of $85 \%$ in patients with median 8 positive lymph nodes by intense dose-dense chemotherapy. As a consequence of the rising numbers of known breast cancer subtypes, 'modern' trial designs become more and more restricted for defined subgroups with the aim of targeted therapy. Trials which recruit estrogen receptor-positive/HER2-negative (ER+/HER-) patients investigate the option of chemotherapy deescalation (PlanB trial) or try to define low-risk groups of ER+/ HER2- patients by short-time neoadjuvant endocrine pre-treatment and its influence on the decline of $\mathrm{Ki}-67$ proliferation (ADAPT trials) [3]. In the past years we made also improvements in defining low-risk ER+/HER2- patients by the additional use of commercially available molecular tests (Mammaprint ${ }^{\circledR}$, OncotypeDX $^{\circledR}$, Endopredict ${ }^{\circledR}$, Prosigna $\left.{ }^{\circledR}\right)$, when all other criteria are inconclusive for therapeutic decision-making. First results of defined low-risk patients in the PlanB and TAILORX trials have shown excellent 3-year and 5-year distant relapse-free survival data with endocrine treatment only $[4,5]$.

On the other hand, we still have a relevant number of high-risk breast cancer patients, where chemotherapy remains standard of care. Even in 2016, tumor burden (number of positive nodes) remains one of the most important risk factors, not only, but especially in patients with $>3$ positive nodes.

If chemotherapy is given to these patients an optimal regimen must be chosen. The Norton-Simon-Hypothesis on log cell kill [6] suggests that chemotherapy should be given at maximum dosages at minimum intervals. Combination chemotherapy, which always has to make compromises regarding the doses of each drug and treatment intervals due to acute as well as cumulative toxicities, does therefore not comply with this theory. Sequential application of monotherapies, however, allows very high single agent doses and dose-dense treatment intervals. Such dose-dense regimens as well as a meta-analysis [7] have shown higher efficacy in comparison to conventionally dosed chemotherapy, but they did not establish a new standard of care, because the published trials reported controversial results. The design of the individual dose-dense trials shows important differences regarding number of cycles, types of drugs and total dose. In addition, some trials are reported as dosedense, but present a mixture of dose-dense and conventional schedules. Also the risk profile of the recruited patients differs remarkably between the trials with a median range of tumor positive lymph nodes between 1 and 8 .

Considering these differences explains why we have negative and positive trials. Although subgroup analyses are considered as hypothesis generating only, they may have consequences for clinical routine. Only (intense) dose-dense trials, which recruited exclusively high-risk patients, showed a significant benefit for ER+ and ER-patients $[8,9]$.

In this issue of BREAST CARE results and therapeutic options of dose-dense-trials in the adjuvant, neoadjuvant, and metastatic situation are discussed.

\section{Disclosure Statement}

V.M. received honoraria from AMGEN, Celgene, and Roche and has an advisory role with Celgene.

\section{KARGER \\ Fax +497614520714




\section{References}

1 Nitz U, Gluz O, Huober J, et al.: Final analysis of the prospective WSG-AGO EC-Doc versus FEC phase II trial in intermediate-risk (pN1) early breast cancer: efficacy and predictive value of Ki67 expression. Ann Oncol 2014;25:1551-1557.

2 Thomssen C, von Minckwitz G, Jackisch C, et al.: Intense dose-dense epirubicin (E), paclitaxel (T), cyclophosphamide (C) (iddETC) is highly effective in adjuvant therapy of node-positive breast cancer patients (pts). An analysis of the GBG, AGO-B, and NOGGO study groups. ESMO 2014, abstr 2570.

3 Hofmann D, Nitz U, Gluz O, et al.: WSG ADAPT adjuvant dynamic marker-adjusted personalized therapy trial optimizing risk assessment and therapy response prediction in early breast cancer: study protocol for a prospective, multi-center, controlled, nonblinded, randomized, investigator initiated phase II/III trial. Trials 2013,14:261
Gluz O, Nitz U, Kreipe HH, et al.: Clinical impact of risk classification by central/local grade or luminal-like subtype vs. Oncotype $\mathrm{DX}^{\circledR}$ : first prospective survival results from the WSG phase III PlanB trial. European Cancer Congress 2015, abst 1937.

5 Sparano JA, Gray RJ, Makower DF, et al.: Prospective validation of a 21 -gene expression assay in breast cancer. N Engl J Med 2015;373:2005-2014.

6 Norton L: A Gompertzian model of human breast cancer growth. Cancer Res 1988;48:7067-7070.

7 Bonilla L, Ben-Aharon I, Vidal L, et al.: Dose-dense chemotherapy in nonmetastatic breast cancer: a systematic review and meta-analysis of randomized controlled trials. J Natl Cancer Inst 2010;102:1845-1854.
Möbus V, Schneeweiss A, du Bois A, et al.: Ten year follow-up analysis of intense dose-dense adjuvant ETC (epirubicin, paclitaxel and cyclophosphamide) confirms superior DFS and OS benefit in comparison to conventional dosed chemotherapy in high-risk breast cancer patients with $>4$ positive lymph nodes. Cancer Res 2012;72(24 suppl): 97s (abstr S3-4).

9 Del Mastro L, De Placido S, Bruzzi P, et al.: Fluorouracil and dose-dense chemo-therapy in adjuvant treatment of patients with early-stage breast cancer: an open-label, $2 \times 2$ factorial, randomised phase 3 trial. Lancet 2015;385:1863-1872. 